OPEN ACCESS

Edited by:

Pietro Scicchitano, ASLBari-Azienda Sanitaria Localedella provincia di Bari (ASL BA), Italy

Reviewed by:

Marco Matteo Ciccone University of Bari Aldo Moro, Italy Gabriella Ricci, ASLBari-Azienda Sanitaria Localedella provincia di Bari (ASL BA), Italy

*Correspondence: Gen-Shan Ma magenshan@hotmail.com Li-Juan Chen

chenlijuan@seu.edu.cn

Specialty section:

This article was submitted to Cardiovascular Epidemiology and Prevention

a section of the journa Frontiers in Cardiovascular Medicine

Received: 25 October 2021 Accepted: 17 January 2022 Published: 21 February 2022

Citation: Qu Y-Y, Zhang X-G, Ju C-W, SU Y-M, Zhang R, Zuo W-J, Ji Z-J, Chen L-J and Ma G-S (2022) Age-Related Utilization of Thrombus Aspiration in Patients With ST-Segment Elevation Myocardial Infarction: Findings From the Improving Care for Cardiovascular Disease in China Project.

Front. Cardiovasc. Med. 9:791007. doi: 10.3389/fCVm.2022.791007

\section{Age-Related Utilization of Thrombus Aspiration in Patients With ST-Segment Elevation Myocardial Infarction: Findings From the Improving Care for Cardiovascular Disease in China Project}

\author{
Yang-Yang Qu, Xiao-Guo Zhang, Cheng-Wei Ju, Ya-Min Su, Rui Zhang, Wen-Jie Zuo, \\ Zhen-Jun Ji, Li-Juan Chen*and Gen-Shan Ma* on behalf of the CCC-ACS Investigators
}

Department of Cardiology, School of Medicine, Zhongda Hospital, Southeast University, Nanjing, China

Background: There are some controversies on the utilization and benefits of thrombus aspiration in patients with ST-segment elevation myocardial infarction (STEMI). However, a few studies investigated this issue and the age-associated effects among the large population in China. Hence, we aimed to figure out the age-associated utilization and in-hospital outcomes of thrombus aspiration to improve therapeutic decisions in clinical routine.

Methods: We retrospectively recruited 13,655 eligible STEMI patients from the database of the Improving Care for Cardiovascular Disease in China-Acute Coronary Syndrome project. These subjects were allocated into primary percutaneous coronary intervention (PPCI)-only group and thrombus aspiration group after being subdivided into three age groups $\left(\mathrm{G}_{21-50}, \mathrm{G}_{51-75}\right.$, and $\left.\mathrm{G}_{76-95}\right)$. After 1:1 propensity score matching for PPCl-only and thrombus aspiration groups, a total of 8,815 matched patients were enrolled for the subsequent analysis. The primary outcome was in-hospital cardiovascular death, and the key safety outcome was in-hospital stroke.

Results: We observed that the ratio of STEMI patients undergoing thrombus aspiration to PPCl-only reduced with aging. For patients $\leq 75$ years, the culprit lesion suffered from thrombus aspiration was mainly located in the left anterior descending branch, and left-ventricular ejection fraction (LVEF) was lower $\left(\mathrm{G}_{21-50}: 54.9 \pm 8.9\right.$ vs. $56.0 \pm 8.7 \%$, $P=0.01 ; G_{51-75}: 53.9 \pm 9.6$ vs. $\left.54.8 \pm 9.0 \%, P=0.001\right)$ and the rate of regional wall motion abnormality was higher $\left(\mathrm{G}_{21-50}: 75.7\right.$ vs. $66.5 \%, P<0.001 ; \mathrm{G}_{51-75}: 75.4$ vs. $69.1 \%, P<0.001$ ) in the thrombus aspiration group. By contrast, for patients $>75$ years, the right coronary artery was the predominant culprit lesion undergoing thrombus aspiration, $\operatorname{LVEF}(63.1 \pm 10.5$ vs. $53.1 \pm 9.5 \%, P=0.985)$ and the regional wall motion abnormality (79.2 vs. $74.2 \%, P=0.089$ ) were comparable between the two treatment groups. Thrombus aspiration neither reduced the in-hospital risk of cardiovascular death, all-cause death, recurrent myocardial infarction, acute stent thrombosis, heart failure, 
cardiogenic shock, and sudden cardiac arrest nor increased stroke risk compared with the PPCl-only group. However, after adjustment for age, thrombus aspiration presented the tendency to reduce the incidence of sudden cardiac arrest ( 4.9 vs. $2.5 \%, P=0.06$ ) and in-hospital cardiovascular death at 3 days (hazard ratio 0.46 ; $95 \% \mathrm{Cl}, 0.20-1.06$; log-rank $P=0.08$ ) in $\mathrm{G}_{76-95}$ group and tended to increase the incidence of heart failure in $\mathrm{G}_{51-75}$ (5.7 vs. 6.9\%, $P=0.07$ ).

Conclusion: The thrombus aspiration neither significantly reduced the in-hospital incidence of major adverse cardiac events nor increased stroke risk. However, it might play a protective role in reducing in-hospital sudden cardiac arrest and increasing survival from cardiovascular death at 3 days for the elderly.

Keywords: thrombus aspiration, primary percutaneous coronary intervention, ST-segment elevation myocardial infarction, age, adverse cardiac events, stroke

\section{BACKGROUND}

ST-segment elevation myocardial infarction (STEMI) is a severe cardiovascular disease, the major cause of which is a complete coronary artery occlusion due to the formation of the thrombus (1). Primary percutaneous coronary intervention (PPCI) within the first $12 \mathrm{~h}$ of the symptom onset has been proven as the most efficient therapeutic means to reperfuse the infarcted myocardium in the clinical routine $(1,2)$. However, the manipulation of atherectomy catheters, balloons, and stents during the PPCI procedure has the chance to cause distal embolization of thrombus. The subsequent obstructed microvasculature, no-reflow phenomenon, impaired tissue perfusion and increased infarct size may lead to cardiovascular death (3).

The routine use of manual thrombus aspiration during the percutaneous coronary intervention (PCI) could improve the primary outcome of microvascular perfusion at 30 days and decrease 1-year cardiac death and non-fatal reinfarction, which was reported by Thrombus Aspiration during Percutaneous Coronary Intervention in Acute Myocardial Infarction Study (TAPAS) $(4,5)$. However, there were some debates on this issue. Thrombus Aspiration in ST-Elevation Myocardial Infarction in Scandinavia (TASTE trial) revealed that thrombus aspiration could not significantly reduce the mortality either at 30 days or 1 year $(6,7)$. As a larger registry enrolling 10,732 patients

\footnotetext{
Abbreviations: ACEI, Angiotensin-converting enzyme inhibitor; AHA, American Heart Association; ARB, Angiotensin receptor blocker; CCC-ACS project, The Improving Care for Cardiovascular Disease in China-Acute Coronary Syndrome project; CI, Confidence interval; CSC, Chinese Society of Cardiology; DAPT, Dual antiplatelet therapy; DES, Drug-eluting stent; GP IIb/IIIa inhibitor, Glycoprotein IIb/IIIa inhibitor; HR, Hazard ratio; LAD, Left anterior descending branch; LCX, Left circumflex branch; LM, Left main coronary artery; LVEF, Left-ventricular ejection fraction; OR, Odds ratio; PCI, Percutaneous coronary intervention; PPCI, Primary percutaneous coronary intervention; RCA, Right coronary artery; SBP, Systolic blood pressure; SD, Standard deviation; STEMI, ST-segment elevation myocardial infarction; TAPAS, The Thrombus Aspiration during Percutaneous Coronary Intervention in Acute Myocardial Infarction Study; TASTE, The Thrombus Aspiration in ST-Elevation Myocardial Infarction in Scandinavia; TOTAL, Trial of Routine Aspiration Thrombectomy with PCI vs. PCI Alone in Patients with STEMI.
}

at 87 hospitals in 20 countries, Trial of Routine Aspiration Thrombectomy with PCI vs. PCI Alone in Patients with STEMI (TOTAL) disclosed that thrombus aspiration could not reduce the 180-day and 1-year risk of cardiovascular death, recurrent myocardial infarction, cardiogenic shock, or heart failure. However, the rates of stroke within 30 and 180 days were higher in the thrombus aspiration group $(2,8)$. Even though thrombus aspiration was not recommended as a routine procedure by TOTAL and TATSE trials, it is considered in case of a large residual thrombus burden $(9,10)$.

To figure out the nationwide utilization and clinical outcomes of thrombus aspiration to provide evidence for therapeutic strategy in clinical routine, we analyzed the real-world data from the Improving Care for Cardiovascular Disease in ChinaAcute Coronary Syndrome (CCC-ACS) project, which is the largest project and quality improvement registry program for ACS in China (11). Considering the utilization of thrombus aspiration differed for patients at different ages, age subgroups were established for the subsequent investigation.

\section{METHODS}

\section{CCC-ACS Project and Data Collection}

CCC-ACS project was co-launched by the American Heart Association (AHA) and Chinese Society of Cardiology (CSC) and collected the information of ACS patients. Unlike TAPAS, TASTE, and TOTAL, which were prospective trials (12-14), the CCC-ACS project did nothing to intervene in the treatment in clinical practice. The principal purpose of this project was to describe the baseline characteristics, in-hospital treatment, and outcomes of ACS patients in China, and then make efforts to optimize the therapeutic strategies and improve therapeutic efficacy accordingly (15). A total of 150 tertiary hospitals from different geographic and economic regions were recruited. The detailed rationale and design of this project have been published before (15). Briefly, in each hospital, the information of the first 20-30 ACS patients was consecutively reported by a welltrained physician on a web-based data collection platform (Oracle Clinical Remote Data Capture; Oracle Corporation, Redwood City, CA, United States) on the official website 
(www.ccc-heart.com) month by month (11). The comprehensive information includes demography, medical history, risk factors, symptoms on arrival, in-hospital laboratory results, reperfusion, medication treatment, and events, as well as medications and counseling at discharge. This project included both unstable angina pectoris and acute myocardial infarction cases, acute myocardial infarction cases have reporting priority. Finally, 63,641 patients diagnosed as the ACS based on the symptoms such as chest pain or distress, alterations of myocardial injury biomarkers, and anomalous results of an electrocardiogram (16, 17) were enrolled from November 2014 to July 2017.

To ensure the accuracy of data collection, the data collection platform was equipped with automatic checks for invalid values. Moreover, the clinical research associates were designated to perform a regular on-site quality inspection. A 5\% of reported cases would be randomly selected for the comparisons between original medical records and reported data. This study was approved by the Ethics Committee of Beijing Anzhen Hospital, Capital Medical University, and it has been registered at www.ClinicalTrials.gov (Unique identifier: NCT02306616).

\section{Recruitment of Study Population}

STEMI was defined according to the guideline issued by the CSC in 2010 (16). In this study, the inclusion criteria of patients were (i) undergoing PPCI within $12 \mathrm{~h}$ of the onset of the symptoms and (ii) placement of at least one drug-eluting stent (DES). The exclusion criteria were (i) placement of a baremetal stent; (ii) subject to coronary artery bypass graft surgery before; (iii) undergoing fibrinolytic therapy at admission; and (iv) lacking information on age, thrombus aspiration treatment (yes / no), or Killip classification. Considering the potential age-associated utilization and dissimilarity of clinical outcomes of thrombus aspiration, the recruited subjects were divided into three age subgroups: $G_{21-50}$ (range $21-50$ years), $G_{51-75}$ (range 51-75 years), and $\mathrm{G}_{76-95}$ (range 76-95 years) for the subsequent analysis.

\section{Study Outcomes}

The occurrence of in-hospital major adverse cardiac cerebrovascular events was evaluated in this study. The primary efficacy outcome was cardiovascular death. The secondary outcomes were all-cause death, recurrent myocardial infarction, acute stent thrombosis, heart failure, cardiogenic shock and sudden cardiac arrest. The key safety outcome was stroke during hospitalization. LV ejection fraction (LVEF) and regional wall motion were examined with echocardiography before discharge.

\section{Statistical Analysis}

Categorical variables were presented as numbers and percentages. Continuous variables were expressed as mean $\pm \mathrm{SD}$ or median (interquartile ranges), as appropriate. A chi-squared test was used to evaluate the differences for categorical variables. Unpaired $t$-test or Mann-Whitney $U$-test was used to test the differences of continuous variables between PPCI-only and thrombus aspiration groups, as applicable. The connection between patients' age and thrombus aspiration treatment was assessed with a logistic regression model and expressed as OR with 95\% CI. All recruited patients were matched between PPCI-only and thrombus aspiration groups with 1:1 propensity score matching in each age subgroup to diminish the influence of confounders. The prespecified covariates used to calculate propensity scores were age, gender, heart rate, systolic blood pressure (SBP), Killip classification, smoking, medical history (prior myocardial infarction, prior PCI, heart failure, hypertension, hyperlipemia, diabetes mellitus, renal failure, cerebral infarction, and cerebral hemorrhage), culprit lesions (left main coronary artery [LM], left anterior descending branch [LAD], left circumflex branch [LCX], right coronary artery [RCA]), in-hospital medications (dual antiplatelet therapy [DAPT, aspirin, and clopidogrel/ticagrelor], angiotensin-converting enzyme inhibitor (ACEI) or angiotensin receptor blocker $[\mathrm{ARB}]$, statin, $\beta$ receptor blocker, glycoprotein IIb/IIIa [GP IIb/IIIa] inhibitor, and low molecular heparin), as well as gross domestic product. The match tolerance was set as 0.02 . For variables with missing data, we imputed the missing values with the sequential regression multiple imputation method implemented by the IVEware software version 0.2 (Survey Research Center, University of Michigan, Ann Arbor, MI, United States). The Cox proportional hazards regression model was used to evaluate time-to-event data of all adverse cardiovascular events after the adjustment of age, and then generate hazard ratio (HR) and 95\% CIs. Kaplan-Meier analysis was performed for assessing survival from in-hospital cardiovascular death at 3, 7, and 10 days, and the statistical significance was tested with the log-rank test. In all cases, two-tailed tests were applied and significance was determined as $P<0.05$.

\section{RESULTS}

\section{Recruitment of Study Population}

A total of 63,641 ACS patients were reported in the database from November 2014 to July 2017, of whom 39,915 (62.7\%) were diagnosed as STEMI; 14,953 of 39,915 (37.5\%) STEMI patients underwent PPCI treatment within $12 \mathrm{~h}$ of the onset of symptoms and implanted at least one DES in the culprit lesion. After excluding patients with coronary artery bypass grafting history, undergoing fibrinolytic therapy at admission, or lacking information on age, thrombus aspiration and Killip classification, 13,655 eligible patients were enrolled for this study (Figure 1).

\section{Age-Associated Utilization of Thrombus Aspiration}

Among the whole enrolled population, the patients in the PPCIonly group were significantly older than those in PPCI combining thrombus aspiration group $(61.2 \pm 12.1$ years vs. $59.7 \pm 12.2$ years, $P<0.001$ ) (Figure 2A). Logistic regression analysis also revealed that thrombus aspiration was less likely to be conducted with the increase of age ( $\mathrm{OR}=0.990 ; 95 \% \mathrm{CI}, 0.987$ to 0.993 ; $P<0.001$ ) (Supplementary Figure 1). We divided the patients into three age subgroups for the subsequent analysis. The patients between 51 and 75 years accounted for a substantial part of the study population $(n=9,097,66.6 \%)$. The proportions of 


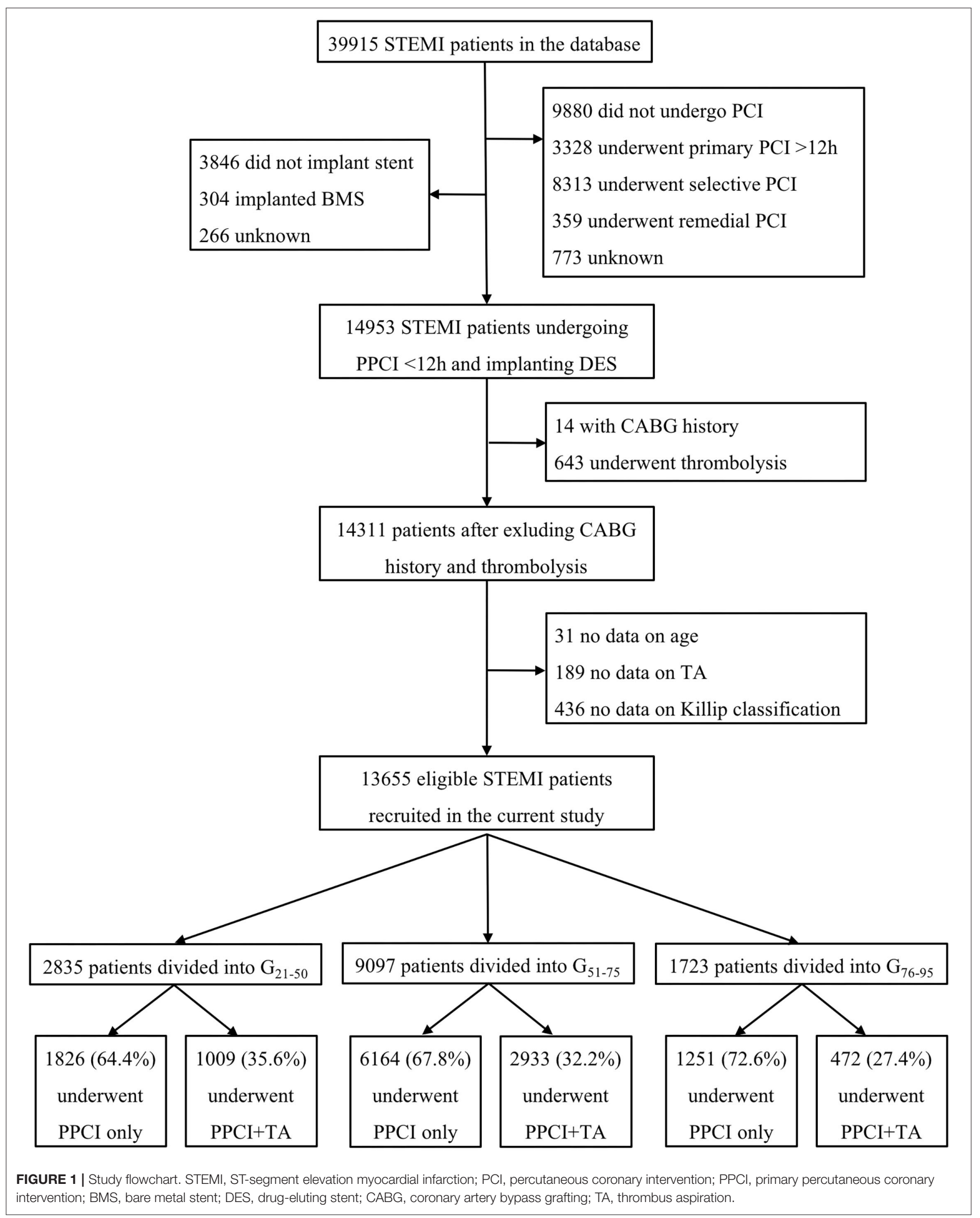



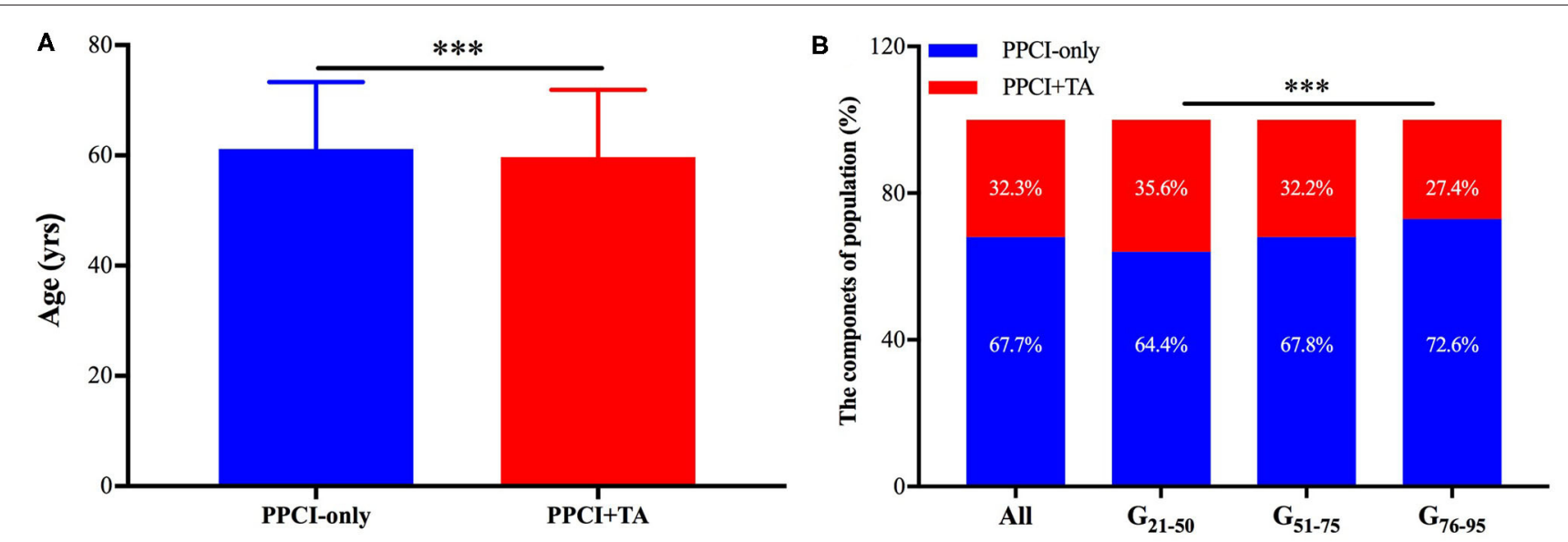

FIGURE 2 | The age-associated utilization of thrombus aspiration. (A) The patients in the PPCl-only group were significantly older than those in PPCI combining thrombus aspiration group. ${ }^{* \star *} P<0.001$. (B) The percentages of STEMI patients undergoing PPCl-only and thrombus aspiration were presented. PPCl, primary percutaneous coronary intervention; TA, thrombus aspiration; $\mathrm{Cl}$, confidence interval.

patients $\leq 50$ years and $>75$ years reached $20.8 \%(n=2,835)$ and $12.6 \%(n=1,723)$, respectively. The percentages of patients undergoing thrombus aspiration in $\mathrm{G}_{21-50}$ and $\mathrm{G}_{51-75}$ groups were discernibly higher than that in the $\mathrm{G}_{76-95}$ group $(35.6 \%$ in $\mathrm{G}_{21-50}, 32.2 \%$ in $\mathrm{G}_{51-75}$, and $27.4 \%$ in $\mathrm{G}_{76-95}, P<0.001$ ), in which the percentage of STEMI patients undergoing PPCI only was almost 1.6 times higher than that of patients undergoing thrombus aspiration (Figure 2B).

\section{Clinical Characteristics of the Study Population}

Age, the proportion of males, body mass index, and heart rate were comparable between PPCI-only and thrombus aspiration groups except for patients in the $\mathrm{G}_{51-75}$ subgroup (age: 62.4 \pm 6.7 years vs. $62.0 \pm 6.8$ years, $P=0.020$ ). The systolic and diastolic blood pressure were higher in the PPCI-only group. The percentage of Killip Class I decreased and the percentage of Killip classes II-IV increased with aging. The most frequently encountered risk factors for the development of STEMI were smoking and hypertension in the whole population. In addition, $\mathrm{G}_{76-95}$ patients showed a lower prevalence of smoking history and hyperlipemia as well as a higher incidence of prior myocardial infarction, PCI history, hypertension, diabetes mellitus and stroke compared with the youngest patients. In the $\mathrm{G}_{21-50}$ subgroup, the medical history was well-balanced between the two groups except for the proportion of smokers $(71.8 \%$ in the PPCI-only group vs. $68.0 \%$ in the thrombus aspiration group, $P=0.036)$ (Table 1).

The culprit lesion differed between PPCI-only and thrombus aspiration groups. Meanwhile, the culprit lesion undergoing thrombus aspiration was more likely to be LAD in $G_{21-50}$ and $\mathrm{G}_{51-75}$ subgroups, and RCA in the $\mathrm{G}_{75-95}$ subgroup. The percentages of patients accepting DAPT, statin, $\beta$ receptor blocker, ACEI or ARB treatment were comparable between PPCIonly and thrombus aspiration groups except for the use of DAPT in the $\mathrm{G}_{76-95}$ group ( 96.6 vs. $98.5 \%, P=0.027$ ). The utilization of anticoagulation therapy including GP IIb/IIIa inhibitor and low molecular heparin increased in the thrombus aspiration group $(P<0.05)$. The hospitalization day was prolonged with the increase of age and remained comparable between PPCI-only and thrombus aspiration groups (Table 2).

\section{Propensity Score Matching for Patients}

After 1:1 propensity score matching for PPCI-only and thrombus aspiration groups, a total of 8,815 matched patients were obtained for the subsequent analysis. The detailed information was presented in Supplementary Tables 1,2. In the $\mathrm{G}_{21-50}$ subgroup, only age $(42.9 \pm 5.3$ years vs. $43.5 \pm 5.8$ years $)$ and the utilization of DAPT (97.7 vs. $98.7 \%$ ) were not well-matched (both $P<0.001$ ). In the $\mathrm{G}_{51-75}$ subgroup, age and Killip class turned out to be comparable after matching. However, because of the possible complexity of characteristics presented in the $\mathrm{G}_{51-75}$ group, there were still differences in blood pressure, culprit lesion, and the utilization of DAPT, statin and anticoagulants. The matching worked excellently in the $G_{76-95}$ subgroup, in which no significant difference of parameters remained between PPCI-only and thrombus aspiration groups.

\section{In-Hospital Clinical Outcomes}

The occurrence of in-hospital adverse cardiovascular events among the matched population was analyzed. The rates of the primary outcome, namely in-hospital cardiovascular death, were $0.5,1.3$ and $4.0 \%$ in each age subgroup, showing no significant difference between PPCI-only and thrombus aspiration groups. Nevertheless, thrombus aspiration reduced the occurrence of cardiovascular death by $27.7 \%$ (Figure 3A). Although the rates of cardiovascular death at 3, 7, and 10 days were comparable between PPCI-only and thrombus aspiration groups in all age subgroups (Figures 3B-D), thrombus aspiration presented the tendency to reduce the occurrence of in-hospital cardiovascular death at 3 days (HR 0.46; 95\% CI, 0.20-1.06; log-rank $P=0.08$ ). Additionally, the rates of secondary outcomes including all-cause 
TABLE 1 | The baseline characteristics of the patients $(n=13,655)$.

\begin{tabular}{|c|c|c|c|c|c|c|c|c|c|}
\hline & \multicolumn{3}{|c|}{$\mathrm{G}_{21-50}$} & \multicolumn{3}{|c|}{$\mathrm{G}_{51-75}$} & \multicolumn{3}{|c|}{$\mathrm{G}_{76-95}$} \\
\hline & $\begin{array}{l}\text { PPCI-only } \\
(n=1,826)\end{array}$ & $\begin{array}{l}\text { PPCI+TA } \\
(n=1,009)\end{array}$ & $P$-value & $\begin{array}{l}\text { PPCI-only } \\
(n=6,164)\end{array}$ & $\begin{array}{c}\text { PPCI+TA } \\
(n=2,933)\end{array}$ & $P$-value & $\begin{array}{l}\text { PPCI-only } \\
(n=1,251)\end{array}$ & $\begin{array}{c}\mathrm{PPCl}+\mathrm{TA} \\
(n=472)\end{array}$ & $P$-value \\
\hline \multicolumn{10}{|l|}{ BASELINE INFORMATION } \\
\hline Age (years) & $44.0 \pm 5.2$ & $43.5 \pm 5.8$ & 0.067 & $62.4 \pm 6.7$ & $62.0 \pm 6.8$ & 0.020 & $80.4 \pm 3.7$ & $80.2 \pm 3.7$ & 0.348 \\
\hline Male, n (\%) & $1,748(95.7 \%)$ & 970 (96.1\%) & 0.602 & 4,959 (80.5\%) & 2,398 (81.8\%) & 0.138 & $780(62.4 \%)$ & 295 (62.5\%) & 0.954 \\
\hline BMI $\left(\mathrm{kg} / \mathrm{m}^{2}\right)$ & $25.4 \pm 3.3$ & $25.2 \pm 3.4$ & 0.351 & $24.4 \pm 3.0$ & $24.5 \pm 2.9$ & 0.111 & $23.4 \pm 3.0$ & $23.5 \pm 3.1$ & 0.653 \\
\hline Heart Rate (bpm) & $81.0 \pm 15.2$ & $81.2 \pm 15.2$ & 0.535 & $77.1 \pm 15.9$ & $76.8 \pm 15.7$ & 0.680 & $76.7 \pm 17.2$ & $76.2 \pm 18.0$ & 0.693 \\
\hline $\mathrm{SBP}(\mathrm{mmHg})$ & $129.0 \pm 22.7$ & $127.0 \pm 22.6$ & 0.019 & $127.6 \pm 23.6$ & $123.9 \pm 23.1$ & $<0.001$ & $126.8 \pm 25.5$ & $123.6 \pm 24.1$ & 0.027 \\
\hline DBP (mmHg) & $82.0 \pm 16.2$ & $80.9 \pm 16.0$ & 0.035 & $78.0 \pm 14.6$ & $76.2 \pm 14.6$ & $<0.001$ & $72.9 \pm 14.7$ & $72.3 \pm 13.8$ & 0.543 \\
\hline \multicolumn{10}{|l|}{ Killip class } \\
\hline I, n (\%) & $1,485(81.3 \%)$ & $843(83.5 \%)$ & 0.155 & 4,752 (77.1\%) & 2,329 (79.4\%) & 0.013 & $866(69.2 \%)$ & $327(69.3 \%)$ & 0.982 \\
\hline II, n (\%) & 266 (14.6\%) & 129 (12.8\%) & 0.189 & $1,070(17.4 \%)$ & 437 (14.9\%) & 0.003 & 274 (21.9\%) & 105 (22.2\%) & 0.878 \\
\hline III, n (\%) & $24(1.3 \%)$ & $13(1.3 \%)$ & 0.958 & 125 (2.0\%) & $49(1.7 \%)$ & 0.245 & $39(3.1 \%)$ & $15(3.2 \%)$ & 0.949 \\
\hline IV, n (\%) & $51(2.8 \%)$ & $24(2.4 \%)$ & 0.510 & 217 (3.5\%) & $118(4.0 \%)$ & 0.234 & $72(5.8 \%)$ & 25 (5.3\%) & 0.713 \\
\hline \multicolumn{10}{|l|}{ MEDICAL HISTORY } \\
\hline Smoking & $1,241(68.0 \%)$ & $724(71.8 \%)$ & 0.036 & $3,188(51.7 \%)$ & 1,542 (52.6\%) & 0.446 & $340(27.2 \%)$ & $130(27.5 \%)$ & 0.880 \\
\hline Prior myocardial infarction & 60 (3.3\%) & 25 (2.5\%) & 0.227 & 268 (4.3\%) & $136(4.6 \%)$ & 0.532 & 59 (4.7\%) & $19(4.0 \%)$ & 0.538 \\
\hline Prior PCl & $59(3.2 \%)$ & $26(2.6 \%)$ & 0.328 & 281 (4.6\%) & $143(4.9 \%)$ & 0.503 & $74(5.9 \%)$ & $23(4.9 \%)$ & 0.402 \\
\hline Hypertension & $668(36.6 \%)$ & 378 (37.5\%) & 0.642 & 3,026 (49.1\%) & $1,474(50.3 \%)$ & 0.299 & 718 (57.4\%) & 267 (56.6\%) & 0.757 \\
\hline Hyperlipemia & $127(7.0 \%)$ & 87 (8.6\%) & 0.108 & 384 (6.2\%) & $212(7.2 \%)$ & 0.072 & 58 (4.6\%) & 27 (5.7\%) & 0.354 \\
\hline Diabetes Mellitus & $236(12.9 \%)$ & $136(13.5 \%)$ & 0.676 & 1,288 (20.9\%) & 599 (20.4\%) & 0.603 & $240(19.2 \%)$ & $88(18.6 \%)$ & 0.799 \\
\hline Stroke & $38(2.1 \%)$ & $20(2.0 \%)$ & 0.859 & $475(7.7 \%)$ & $258(8.8 \%)$ & 0.074 & 149 (11.9\%) & $61(12.9 \%)$ & 0.566 \\
\hline
\end{tabular}

Results are reported as mean $\pm S D$ or $n(\%)$. PPCl, primary percutaneous coronary intervention; TA, thrombus aspiration; BMI, body mass index; SBP, systolic blood pressure; DBP, diastolic blood pressure. The bold values indicate that the values smaller than 0.5 implying significant difference. 


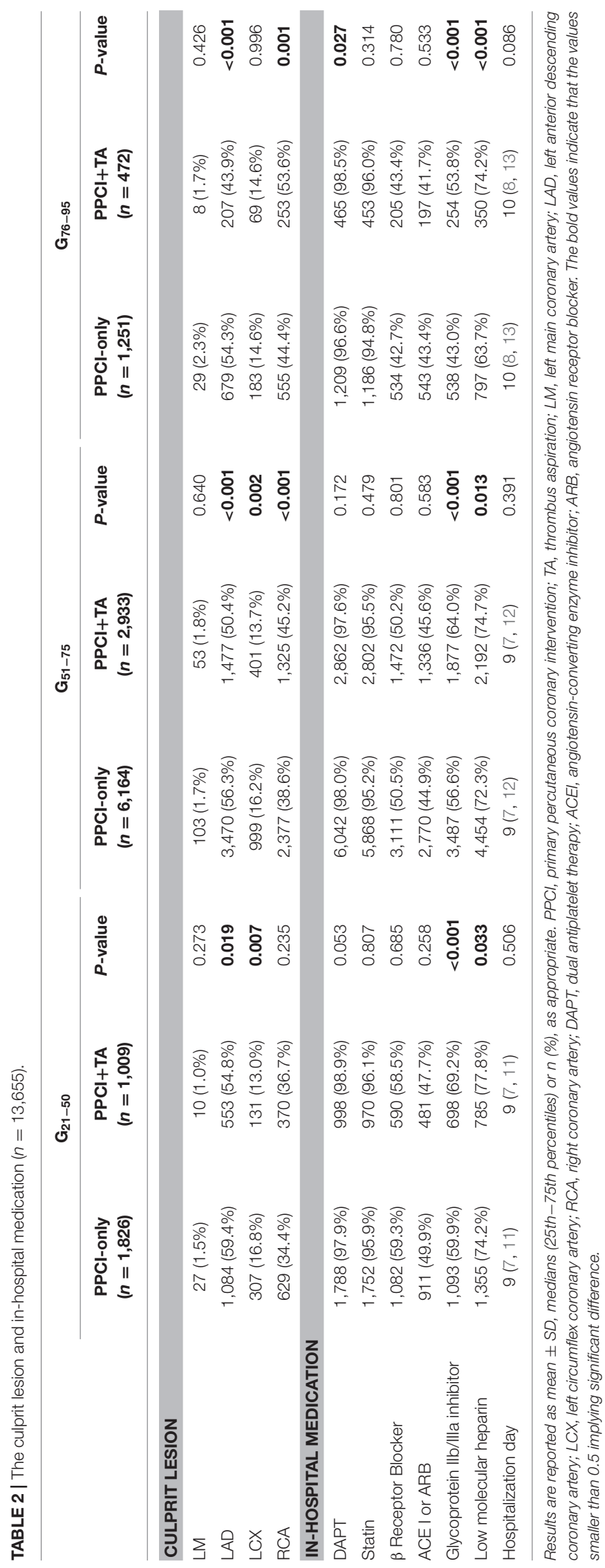

death, recurrent myocardial infarction, acute stent thrombosis, heart failure, cardiogenic shock, sudden cardiac arrest and stroke were comparable between two treatment groups in three age subgroups (all $P>0.05$ after adjustment for age, Figure 4). While, in the $\mathrm{G}_{51-75}$ group, we observed a marginally higher rate of heart failure in patients undergoing thrombus aspiration (5.7 vs. $6.9 \%$, adjusted $P=0.07$ ). Meanwhile, the rate of sudden cardiac arrest was insignificantly lower in the thrombus aspiration group ( 4.9 vs. $2.5 \%$, adjusted $P=0.06$ ) for the elderly.

Stroke was regarded as the key safety outcome, of which the incidence increased with aging and sustained the similarity between PPCI-only and thrombus aspiration groups (all $P>$ 0.05 , Figure 5).

The last echocardiography examination before discharge presented that among patients $\leq 75$ years old, LVEF was lower and the rate of regional wall motion abnormality was higher in the thrombus aspiration group (both $P<0.05$ ), while no significant difference was observed in the $\mathrm{G}_{76-95}$ subgroup. Also, the incidence of regional wall motion abnormality raised with the increase of age (Supplementary Figure 2).

\section{DISCUSSION}

To the best of our knowledge, it is the largest nationwide, multicenter, retrospective epidemiological study to evaluate the age-associated utilization and clinical outcomes of thrombus aspiration among STEMI patients undergoing PPCI within $12 \mathrm{~h}$ of symptoms onset and implanting DES in China. We demonstrated that thrombus aspiration did not increase the rate of in-hospital major adverse cardiac cerebrovascular events (e.g., cardiovascular death and stroke). However, the utilization of thrombus aspiration in patients older than 75 years helped maintain LVEF and regional wall motion normality and presented the tendency toward diminishing the risk of in-hospital cardiovascular death at 3 days and sudden cardiac arrest.

According to the data from the CCC-ACS database, thrombus aspiration is still being used in a substantial number of STEMI patients. A $32.3 \%$ the STEMI patients undergoing PPCI and implanting DES were treated with upfront or bailout thrombus aspiration. STEMI patients were less likely to be treated with thrombus aspiration with the increase of age. We found that the most frequently encountered risk factor of STEMI was smoking in patients $\leq 75$ years and hypertension in patients $>75$ years, this phenomenon was partially in line with the previous findings $(18,19)$. For patients aged $21-50$ years, the percentage of smokers was higher in the thrombus aspiration group.

In the entire population, the incidences of in-hospital adverse cardiovascular events were comparable between PPCI-only and thrombus aspiration groups. This finding was sort of consistent with the results in TASTE, TOTAL and a large observational study $(n=42,829)$ using available data from the Swedish Coronary Angiography and Angioplasty Registry (SCAAR) at 30 days $(7,8,20)$. Due to the ventricular remodeling, diastolic dysfunction and decreased vascular compliance with aging, the elderly presented higher mortality when suffering from the sudden occlusion of the coronary artery (21). Nevertheless, we 


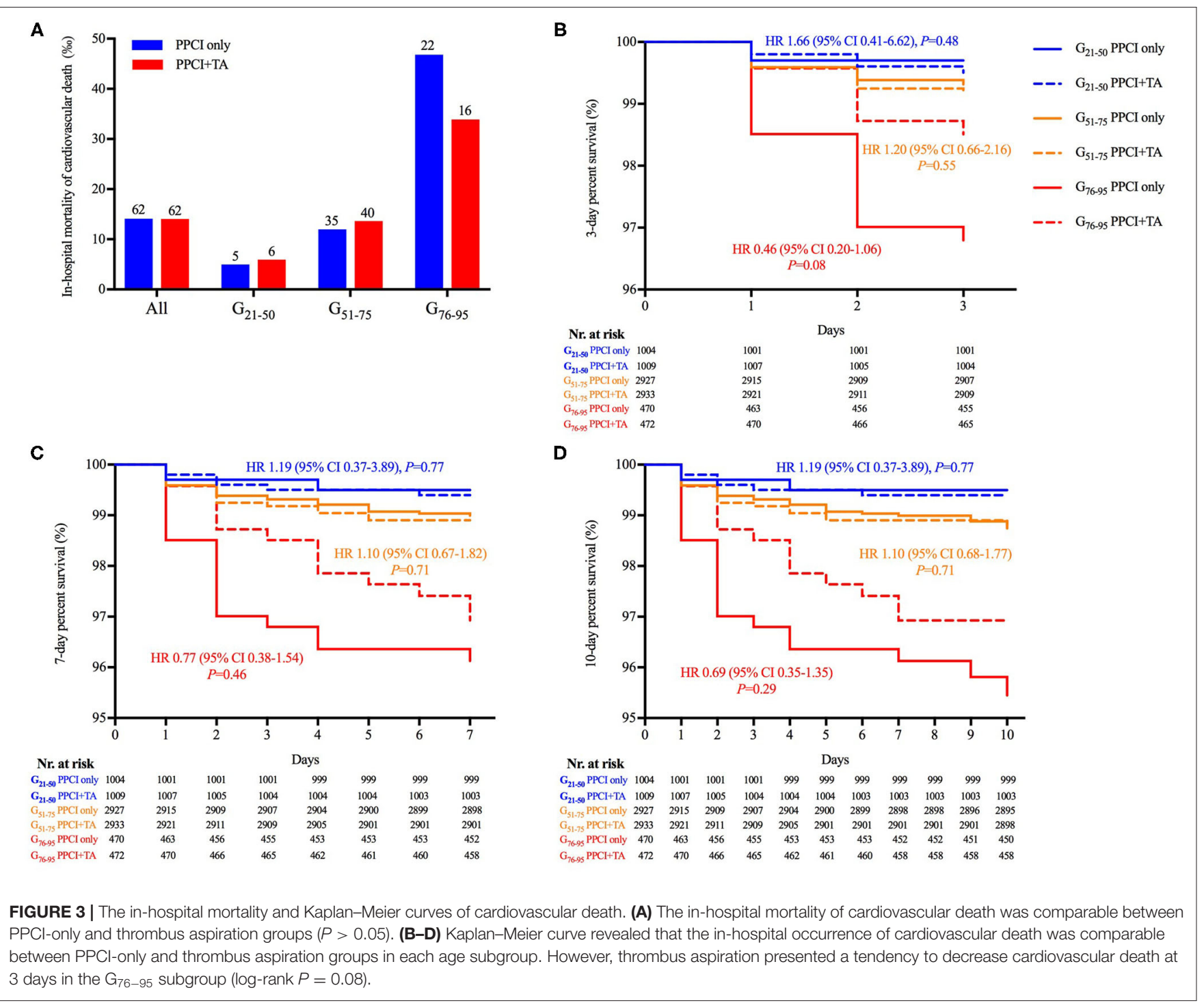

detected that thrombus aspiration tended to reduce the risk of in-hospital cardiovascular death at 3 days by $27.6 \%$ in the $\mathrm{G}_{76-95}$ group. It is noteworthy that thrombus aspiration also tended to decline the rate of rehospitalization due to reinfarction ( 0.5 vs. $0.9 \%$; HR $0.61 ; 95 \%$ CI, $0.34-1.07 ; P=0.09$ ) and stent thrombosis ( 0.2 vs. $0.5 \%$; HR $0.47 ; 95 \% \mathrm{CI}, 0.20-1.02 ; P=0.06$ ) at 30 days in TASTE (7). Meanwhile, we noticed that the incidence of stent thrombosis in the PPCI-only group was 1.5-3 times compared to that in the thrombus aspiration group, although the difference was insignificant. Considering the extremely low incidence of stent thrombosis, whether thrombus aspiration can reduce the occurrence of this event needs to be further investigated in a larger population. What is more, thrombus aspiration showed a protective impact on reducing the prevalence of sudden cardiac death in the elderly ( 4.9 vs. $2.5 \%, P=0.06$ ). While, in the $\mathrm{G}_{51-75}$ group, we observed a higher rate of heart failure in patients undergoing thrombus aspiration, which may raise physicians' attention in the clinical practice.
A part of the information derived from angiography and the last echocardiography before discharge was recorded in the CCC-ACS database. Among patients $\leq 75$ years, the culprit lesion suffered from thrombus aspiration was mainly located in LAD, LVEF was lower and the rate of regional wall motion abnormality was higher in the thrombus aspiration group. STEMI patients with a high thrombus burden are more likely to benefit from thrombus aspiration compared with patients with a low thrombus burden, suggesting the more frequent utilization of thrombus aspiration in patients with high thrombus burden (10). Ahmed et al. uncovered that the higher thrombus grade was associated with larger infarct size and slightly worse LV function (22). Hence, we hypothesized that the deterioration of cardiac function and myocardial wall motion might be associated with the burden of coronary thrombus rather than the treatment. Unfortunately, the severe impairment of cardiac performance cannot be simply renovated by thrombus aspiration in a short time. By contrast, among patients $>75$ years, the culprit lesion 


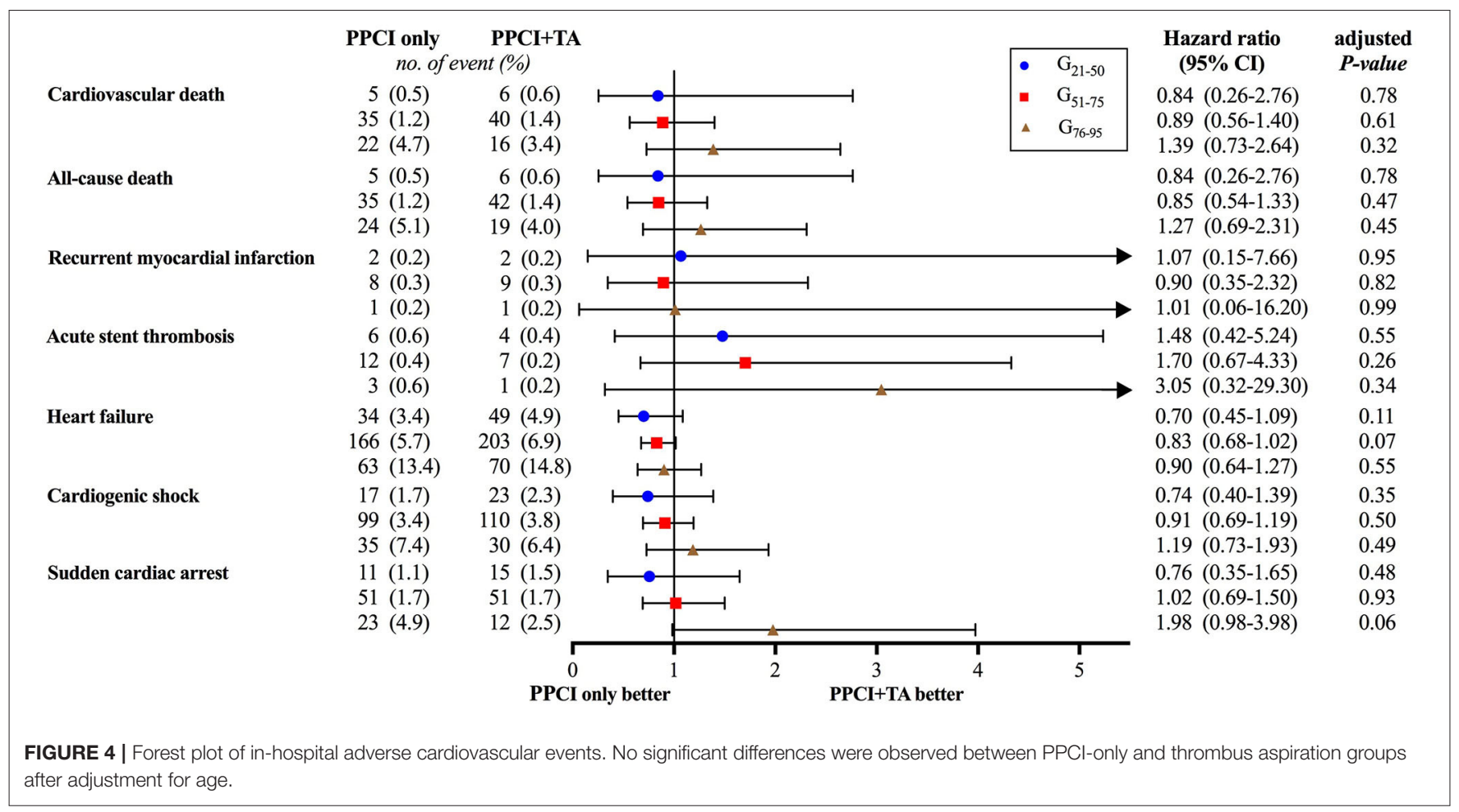

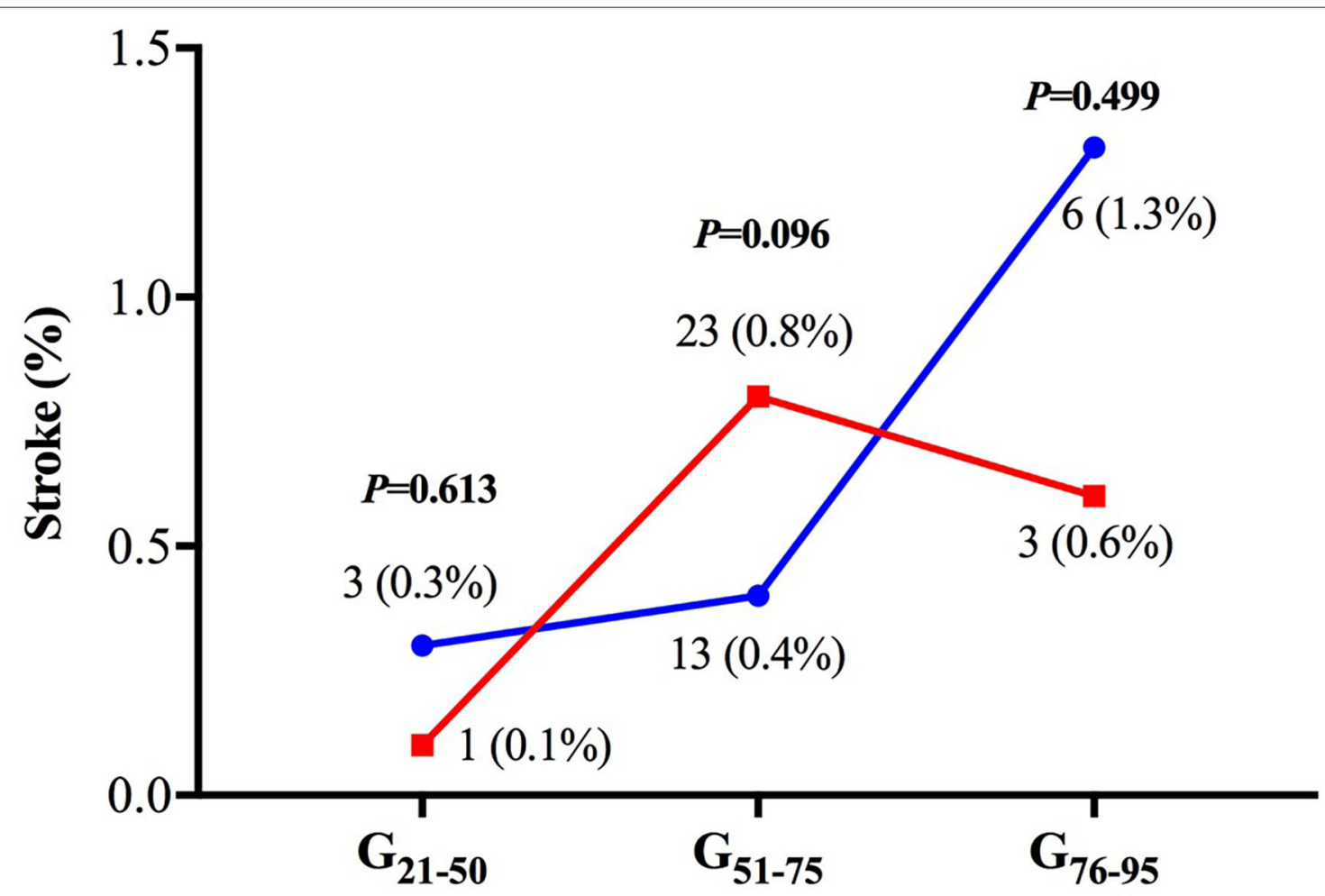

FIGURE 5 | The incidence of in-hospital stroke. There was no significant difference in the risk of in-hospital stroke between the two treatment groups in all age subgroups. 
suffered from thrombus aspiration was mainly located in RCA, and LVEF and the incidence of regional wall motion abnormality were comparable between the two treatment groups. Unlike LAD predominantly supplying blood to the anterior and septal proportions of LV myocardium, RCA principally supplies the right ventricle and atrium (23). Meanwhile, the elderly patients suffering from persistent angina have robust collateral circulation to restore flow, which might help remain the blood supply and cardiac function to some extent (24).

There are still debates on whether thrombus aspiration can increase the rate of stroke. In this study, we did not find significantly increased in-hospital occurrence of the stroke in the thrombus aspiration group, which was similar in TASTE and other research studies $(7,20)$. Although TOTAL propagated the attitude that thrombus aspiration could increase the occurrence of a stroke at 30 and 180 days ( $P=0.020$ and 0.002 , respectively), the conclusion was drawn among the randomized population with crossover from their initial treatment allocation to the alternative therapy in both groups. Looking back on the ontreatment analysis among patients who received upfront or bailout thrombus aspiration irrespective of randomization and convention PCI in TOTAL, the thrombus aspiration could not increase the rate of stroke at 30 and 180 days $(P=0.193$ and 0.068 , respectively) (8). TOTAL also indicated that the application of thrombus aspiration among patients with high thrombus burden (thrombolysis in myocardial infarction [TIMI] thrombus Grade $\geq 3$ ) rather than those with low thrombus burden could increase the rate of stroke at 30 days $(0.7 \%$ in thrombus aspiration group vs. $0.4 \%$ in PCI-only group; $\mathrm{HR}=$ 1.90; 95\% CI, 1.04-3.08; $P=0.03$ ) (25). In the TOTAL trial, the minimal volume of thrombus aspiration procedures required for operators only reached five in the last 2 years (14). The utilization of upfront bailout GP IIb/IIIa inhibitor was more frequent among the patients undergoing thrombus aspiration treatment than those in the PCI-only group (25). Hence, we cannot rule out that the higher rate of stroke was caused by procedures during thrombus aspiration therapy or more intensive anticoagulation therapy after thrombus aspiration. It is a consensus that the inappropriate operation of thrombus aspiration devices may cause systemic embolization of thrombotic material or air embolism (26). So, the development of aspiration catheters and improvement of thrombus aspiration skills need to be emphasized to avoid the increased ischemic stroke risk, especially among patients with a high thrombus burden. Meanwhile, the patients with a high thrombus burden are more likely to suffer from hemorrhagic stroke due to the intensive anticoagulants and need to be closely monitored in intensive or cardiac care units post procedurally.

\section{LIMITATIONS}

There were several limitations in this study. First, the CCCACS project is a retrospective and observational real-world study based on the medical records uploaded in the database, limited data were gathered. Hence, the detailed information of properties of thrombus (red/white), location of thrombus (proximal/distal),
TIMI thrombus grade, size and length of the stent, bifurcation, collateral circulation and so on were inaccessible. However, this study still provides physicians with a better understanding of the utilization and clinical benefit of thrombus aspiration. Second, due to the numerous factors considered, there were still differences with the potential confounders between PPCIonly and thrombus aspiration groups after 1:1 propensity score matching. While, most variables were well-matched, the difference of age in the unmatched population was taken into consideration during the analysis of clinical outcomes to increase the reliability of results.

\section{CONCLUSION}

In this large nationwide observational study, thrombus aspiration did not significantly reduce the in-hospital risk of cardiovascular death, all-cause death, recurrent myocardial infarction, acute stent thrombosis, heart failure, cardiogenic shock, sudden cardiac arrest and stroke compared with conventional PPCI among the whole STEMI patients. However, it presented the tendency to reduce the occurrence of in-hospital cardiovascular death at 3 days and sudden cardiac arrest among patients older than 75 years, and increase the risk of heart failure in patients aged 51-75 years.

\section{DATA AVAILABILITY STATEMENT}

The datasets presented in this article are not readily available because the datasets analyzed in this study are not publicly available according to the regulation of CCC-ACS project, but the reliability and accuracy can be validated with the assistance of Prof. Dong Zhao who is the principle investigator of this project. Requests to access the datasets should be directed to Prof. Dong Zhao,deezhao@vip.sina.com.

\section{ETHICS STATEMENT}

The studies involving human participants were reviewed and approved by the Beijing Anzhen Hospital. Written informed consent for participation was not required for this study in accordance with the national legislation and the institutional requirements.

\section{AUTHOR CONTRIBUTIONS}

G-SM and L-JC contributed to the design and overall investigation. Y-YQ was responsible for the data collection, statistical analysis, and manuscript. X-GZ, C-WJ, Y-MS, RZ, $\mathrm{W}-J \mathrm{Z}$, and Z-JJ have made substantial contributions to the analysis and interpretation of data or revising the manuscript. All authors read and approved the final manuscript.

\section{FUNDING}

CCC-ACS project was a collaborative study co-launched by the American Heart Association (AHA) and the Chinese Society 
of Cardiology (CSC). The AHA was funded by Pfizer and AstraZeneca for the quality improvement initiative through an independent grant for learning and change.

\section{ACKNOWLEDGMENTS}

We acknowledge the contribution of all investigators, including the physicians participating in the project from 150 hospitals (Supplementary Table 3).

\section{SUPPLEMENTARY MATERIAL}

The Supplementary Material for this article can be found online at: https://www.frontiersin.org/articles/10.3389/fcvm. 2022.791007/full\#supplementary-material

\section{REFERENCES}

1. Anderson JL, Morrow DA. Acute myocardial infarction. N Engl J Med. (2017) 376:2053-64. doi: 10.1056/NEJMra1606915

2. Jolly SS, Cairns JA, Yusuf S, Rokoss MJ, Gao P, Meeks B, et al. Outcomes after thrombus aspiration for ST elevation myocardial infarction: 1-year followup of the prospective randomised TOTAL trial. Lancet. (2016) 387:12735. doi: 10.1016/S0140-6736(15)00448-1

3. Ge J, Li J, Dong B, Ning X, Hou B. Determinants of angiographic thrombus burden and impact of thrombus aspiration on outcome in young patients with ST-segment elevation myocardial infarction. Catheter Cardiovasc Interv. (2019) 93:E269-76. doi: 10.1002/ccd.27944

4. Svilaas T, Vlaar PJ, van der Horst IC, Diercks GF, de Smet BJ, van den Heuvel AF, et al. Thrombus aspiration during primary percutaneous coronary intervention. N Engl J Med. (2008) 358:557-67. doi: 10.1056/NEJMoa0706416

5. Vlaar PJ, Svilaas T, van der Horst IC, Diercks GF, Fokkema ML, de Smet BJ, et al. Cardiac death and reinfarction after 1 year in the Thrombus Aspiration during Percutaneous coronary intervention in Acute myocardial infarction Study (TAPAS): a 1-year follow-up study. Lancet. (2008) 371:191520. doi: 10.1016/S0140-6736(08)60833-8

6. Lagerqvist B, Frobert O, Olivecrona GK, Gudnason T, Maeng M, Alstrom P, et al. Outcomes 1 year after thrombus aspiration for myocardial infarction. $N$ Engl J Med. (2014) 371:1111-20. doi: 10.1056/NEJMoa1405707

7. Frobert O, Lagerqvist B, Olivecrona GK, Omerovic E, Gudnason T, Maeng $\mathrm{M}$, et al. Thrombus aspiration during ST-segment elevation myocardial infarction. N Engl J Med. (2013) 369:1587-97. doi: 10.1056/NEJMoa1308789

8. Jolly SS, Cairns JA, Yusuf S, Meeks B, Pogue J, Rokoss MJ, et al. Randomized trial of primary PCI with or without routine manual thrombectomy. New Engl J Med. (2015) 372:1389-98. doi: 10.1056/NEJMoa1415098

9. Ibanez B, James S, Agewall S, Antunes MJ, Bucciarelli-Ducci C, Bueno H, et al. 2017 ESC Guidelines for the management of acute myocardial infarction in patients presenting with ST-segment elevation: The Task Force for the management of acute myocardial infarction in patients presenting with STsegment elevation of the European Society of Cardiology (ESC). Eur Heart J. (2018) 39:119-77. doi: 10.1093/eurheartj/ehx393

10. O'Gara PT, Kushner FG, Ascheim DD, Casey DE, Chung MK, de Lemos JA, et al. 2013 ACCF/AHA guideline for the management of ST-elevation myocardial infarction: a report of the American College of Cardiology Foundation/American Heart Association Task Force on Practice Guidelines. J Am Coll Cardiol. (2013) 61:e78-140. doi: 10.1016/j.jacc.2012.11.019

11. Hao Y, Liu J, Liu J, Yang N, Smith SC, Jr., et al. Sex differences in in-hospital management and outcomes of patients with acute coronary syndrome. Circulation. (2019) 139:177685. doi: 10.1161/CIRCULATIONAHA.118.037655

12. Svilaas T, van der Horst IC, Zijlstra F. Thrombus aspiration during percutaneous coronary intervention in Acute myocardial infarction Study (TAPAS)-study design. Am Heart J. (2006) 151:597.e1-e7. doi: 10.1016/j.ahj.2005.11.010
Supplementary Figure 1 | Logistic regression analysis revealed that thrombus aspiration was less likely to be conducted with the increase of age. PPCl, primary percutaneous coronary intervention; TA, thrombus aspiration; $\mathrm{Cl}$, confidence interval.

Supplementary Figure 2 | LVEF and the rate of region wall motion abnormality before discharge. (A,B) For patients $\leq 75$ years, patients undergoing thrombus aspiration treatment presented with lower LVEF and a higher rate of regional wall motion abnormality. While for patients $>75$ years, no significant difference was observed between the two treatment groups. RWMA, regional wall motion abnormality.

Supplementary Table 1 | The baseline characteristics of the matched patients $(n=8,815)$.

Supplementary Table 2 | The culprit lesion and in-hospital medication of the matched patients $(n=8,815)$.

Supplementary Table 3 | One hundred and fifty hospitals and investigators participating in the CCC-ACS project during 2014-2017.

13. Frobert O, Lagerqvist B, Gudnason T, Thuesen L, Svensson R, Olivecrona GK, et al. Thrombus Aspiration in ST-Elevation myocardial infarction in Scandinavia (TASTE trial). A multicenter, prospective, randomized, controlled clinical registry trial based on the Swedish angiography and angioplasty registry (SCAAR) platform. Study design and rationale. Am Heart J. (2010) 160:1042-8. doi: 10.1016/j.ahj.2010.08.040

14. Jolly SS, Cairns J, Yusuf S, Meeks B, Shestakovska O, Thabane L, et al. Design and rationale of the TOTAL trial: a randomized trial of routine aspiration ThrOmbecTomy with percutaneous coronary intervention (PCI) versus PCI ALone in patients with ST-elevation myocardial infarction undergoing primary PCI. Am Heart J. (2014) 167:315-21.e1. doi: 10.1016/j.ahj.2013.12.002

15. Hao Y, Liu J, Liu J, Smith SC, Huo Y. Rationale and design of the Improving Care for Cardiovascular Disease in China (CCC) project: A national effort to prompt quality enhancement for acute coronary syndrome. Am Heart J. (2016) 179:107-15. doi: 10.1016/j.ahj.2016.06.005

16. China Society of Cardiology of Chinese Medical A, Editorial Board of Chinese Journal of C. [Guideline for diagnosis and treatment of patients with STelevation myocardial infarction]. Zhonghua Xin Xue Guan Bing Za Zhi. (2010) 38:675-90. doi: 10.3760/cma.j.issn.0253-3758.2010.08.002

17. Chinese Society of Cardiology of Chinese Medical A, Editorial Board of Chinese Journal of C. [Guideline of non-ST segment elevation acute coronary syndrome]. Zhonghua Xin Xue Guan Bing Za Zhi. (2012) 40:353-67. doi: 10.3760/cma.j.issn.0253-3758.2012.05.001

18. Puricel S, Lehner C, Oberhansli M, Rutz T, Togni M, Stadelmann M, et al. Acute coronary syndrome in patients younger than 30 years - aetiologies, baseline characteristics and long-term clinical outcome. Swiss Med Wkly. (2013) 143:13816. doi: 10.4414/smw.2013.13816

19. Tong J, Xiang WW, Ang AS, Sim WJ, Quah KH, Foo D, et al. Clinical outcomes of elderly South-East Asian patients in primary percutaneous coronary intervention for ST-elevation myocardial infarction. $J$ Geriatr Cardiol. (2016) 13:830-5. doi: 10.11909/j.issn.1671-5411.2016.10.001

20. Angeras O, Haraldsson I, Redfors B, Frobert O, Petursson P, Albertsson P, et al. Impact of thrombus aspiration on mortality, stent thrombosis, and stroke in patients with ST-segment-elevation myocardial infarction: a report from the swedish coronary angiography and angioplasty registry. J Am Heart Assoc. (2018) 7:7680. doi: 10.1161/JAHA.117.007680

21. Gao L, Hu X, Liu YQ, Xue Q, Feng QZ. Percutaneous coronary intervention in the elderly with ST-segment elevation myocardial infarction. Clin Interv Aging. (2014) 9:1241-6. doi: 10.2147/CIA.S62642

22. Ahmed TA, Sorgdrager BJ, Cannegieter SC, van der Laarse A, Schalij MJ, Jukema W. Pre-infarction angina predicts thrombus burden in patients admitted for ST-segment elevation myocardial infarction. EuroIntervention. (2012) 7:1396-405. doi: 10.4244/EIJV7I12A219

23. Rehman I, Rehman A. Anatomy, Thorax, Heart Left Anterior Descending (LAD) Artery. Treasure Island, FL: StatPearls (2019).

24. Epstein SE, Lassance-Soares RM, Faber JE, Burnett MS. Effects of aging on the collateral circulation, and therapeutic implications. 
Circulation. (2012) 125:3211-9. doi: 10.1161/CIRCULATIONAHA.111. 079038

25. Jolly SS, Cairns JA, Lavi S, Cantor WJ, Bernat I, Cheema AN, et al. Thrombus aspiration in patients with high thrombus burden in the TOTAL trial. J Am Coll Cardiol. (2018) 72:1589-96. doi: 10.1016/j.jacc.2018. 07.047

26. Mahmoud KD, Zijlstra F. Thrombus aspiration in acute myocardial infarction. Nat Rev Cardiol. (2016) 13:418-28. doi: 10.1038/nrcardio. 2016.38

Conflict of Interest: The authors declare that the research was conducted in the absence of any commercial or financial relationships that could be construed as a potential conflict of interest.
Publisher's Note: All claims expressed in this article are solely those of the authors and do not necessarily represent those of their affiliated organizations, or those of the publisher, the editors and the reviewers. Any product that may be evaluated in this article, or claim that may be made by its manufacturer, is not guaranteed or endorsed by the publisher.

Copyright ( 2022 Qu, Zhang, Ju, Su, Zhang, Zuo, Ji, Chen and Ma. This is an open-access article distributed under the terms of the Creative Commons Attribution License (CC BY). The use, distribution or reproduction in other forums is permitted, provided the original author(s) and the copyright owner(s) are credited and that the original publication in this journal is cited, in accordance with accepted academic practice. No use, distribution or reproduction is permitted which does not comply with these terms. 